\title{
Movement Kinematics and Cortical Activation in Children with and without Autism Spectrum Disorder During Sway Synchrony Tasks: An fNIRS Study
}

\author{
Wan-Chun Su \\ University of Delaware \\ McKenzie Culotta \\ University of Delaware \\ Daisuke Tsuzuki \\ Tokyo Metropolitan University \\ Anjana Bhat ( $\sim$ abhat@udel.edu ) \\ University of Delaware
}

\section{Research Article}

Keywords:

Posted Date: March 11th, 2021

DOl: https://doi.org/10.21203/rs.3.rs-268551/v1

License: (c) (i) This work is licensed under a Creative Commons Attribution 4.0 International License.

Read Full License

Version of Record: A version of this preprint was published at Scientific Reports on July 22nd, 2021. See the published version at https://doi.org/10.1038/s41598-021-94519-4. 


\title{
Movement Kinematics and Cortical Activation in Children with and without Autism Spectrum Disorder During Sway Synchrony Tasks: An fNIRS Study
}

\author{
Wan-Chun Su, ${ }^{1,2}$ McKenzie Culotta, ${ }^{1,2}$ Daisuke Tsuzuki, ${ }^{3}$ Anjana Bhat, ${ }^{1,2,4 *}$ \\ ${ }^{1}$ Department of Physical Therapy, University of Delaware, Newark, DE \\ ${ }^{2}$ Biomechanics and Movement Science Program, University of Delaware, Newark, DE \\ ${ }^{3}$ Department of Language Sciences, Tokyo Metropolitan University, Tokyo, Japan \\ ${ }^{4}$ Department of Psychological and Brain Sciences, University of Delaware, Newark, DE \\ *Corresponding author: Anjana Bhat \\ Postal address: 540 S College Avenue, University of Delaware, Newark, Delaware, United States of America \\ E-mail: abhat@udel.edu \\ Phone: 443-523-8680
}




\begin{abstract}
Children with Autism Spectrum Disorder (ASD) have difficulties with socially embedded movements such as imitation and interpersonal synchrony (IPS); however, related movement characteristics and underlying neural mechanisms are not well understood.

Aim: This study compared the movement characteristics and cortical activation patterns of children with and without ASD during a whole-body, sway synchrony task when different levels of social information were provided.

Methods: Thirty children with and without ASD (mean age: 12.6 years, SE: 0.6 years) participated. Movement kinematics and fNIRS-based cortical activation were recorded when the child observed an adult tester sway side to side, when they swayed solo, or when they swayed face to face with the tester with or without fingertips touching (i.e., IPS).

Results: Children with ASD showed reduced synchrony and insufficient sway amplitude compared to typically developing children without ASD. They showed reduced cortical activation over the inferior frontal gyrus and superior temporal sulcus during IPS and failed to increase their cortical activation when more social information was provided. The cortical activation findings were significantly associated with IPS behaviors and social communication performance.

Conclusion: The ASD-related neurobiomarkers identified in our study could be used as objective measures to evaluate intervention effects in children with ASD.
\end{abstract}




\section{Introduction}

Autism Spectrum Disorder (ASD) is a highly prevalent neurodevelopmental disorder affecting 1 in 59 children in the US. ${ }^{1}$ Children with ASD present with diagnostic symptoms of impaired social communication skills (DSM-5) ${ }^{2}$ as well as perceptuo-motor comorbidities which affects their ability to move with others within social contexts. ${ }^{3}$ For example, children with ASD might have impaired social monitoring and poor motor planning/incoordination that in turn affects their ability to imitate (i.e., copy discrete actions) ${ }^{4}$ or synchronize continuous actions with others, also known as interpersonal synchrony (IPS) ${ }^{5,6}$ During infancy and early childhood, infants and children learn a variety of social communication skills from their social partners by engaging in imitation and IPS. ${ }^{7,8}$ These interactions help facilitate children's relationships with their caregivers and peers. ${ }^{9,10}$ Difficulties in engaging in imitation and IPS will affect the social cognitive development of children with ASD and will limit their opportunities to build relationships with other social partners. ${ }^{11}$

Studies have reported reduced IPS and atypical movement characteristics including slower movement speed and inaccurate movements in children with ASD., ${ }^{5,12}$ The neuroimaging studies also suggested atypical activation over the frontal, temporal and parietal lobes when children with ASD performed imitation and IPS tasks. ${ }^{13,14}$ However, the neuroimaging studies were limited to finger motions or seated reaching tasks that did not involve whole-body movements or use of objective kinematic measures using motion tracking. Moreover, changing levels of social information (e.g., social-visual or social-tactile) could variably influence IPS behaviors and corresponding cortical activation. Using functional near-infrared spectroscopy (fNIRS), a novel neuroimaging tool that is robust against movement artifacts, the present study compared the movement characteristics and associated cortical activation in children with and without ASD 
during a whole-body sway synchrony task and investigated how different levels of social information might alter IPS behaviors and associated cortical activation.

\section{IPS behaviors and associated cortical activation}

IPS is an important everyday activity that requires complex social and perceptuo-motor skills. Some example of IPS include people walking at the same pace or dancing with a partner. ${ }^{15,16}$ When engaging in IPS, one needs to understand the shared goal of the task as well as one's own individual role in the task. ${ }^{17}$ For example, when dancing with a partner, the common goal is to move in harmony, while the individual goals might focus on moving limbs and shifting weight in a certain direction. Additionally, partners need to perceive and monitor the information from the environment (e.g., the locations of the obstacles in the room), and based on their own and their partner's actions (e.g., eye gaze, body posture, etc.). ${ }^{17}$ The environmental and social information will help in anticipating/predicting the partner's movements and reactively adjusting one's own movements to keep up with those of the partner's. ${ }^{17}$ During synchronization, one might perceive faster or slower movements of their partner and plan ahead or reactively change the speed of their own movements to stay in synchrony with the partner. The monitoring and adjustments of actions during IPS requires continuous perceptuo-motor integration which is also needed during imitation and other joint actions. ${ }^{17}$

Several cortical regions in bilateral frontal, parietal, and temporo-occipital lobes that support imitation behaviors may also play a role in IPS. ${ }^{18}$ The inferior frontal gyrus (IFG) plays a role in encoding the goal of actions as it is more active when performing goal-directed than non-goal directed movements. ${ }^{19}$ It also plays a role in perceiving the salience of action information offered by partners and in inferring intentions of actions. ${ }^{20}$ Furthermore, fronto-parietal connections are important in integrating multisensory information from the environment and social partners. ${ }^{21}$ The 
IFG and inferior parietal lobe (IPL) interact with each other and are important for matching observed actions with internal motor plans and anticipatory/predictive control of actions..$^{22,23}$ The superior temporal sulcus (STS) plays an important role in establishing visuomotor correspondence and is activated as one monitors and matches their own actions to those of their partner's in a predictive or reactive manner. ${ }^{24}$ In fact, previous fMRI studies found that IFG, STS, and IPL regions are important parts of the imitation network and are more active during imitation compared to its component behaviors, including action observation and execution. ${ }^{25}$ Other important brain regions for IPS include pre- and post-central gyrus (PCG) and the prefrontal regions (including middle frontal gyrus (MFG)). PCG includes the primary motor and somatosensory cortices that interact with other cortical regions to receive/process sensory information and execute actions. ${ }^{26}$ The MFG along with other prefrontal regions are important for executive functions such as motor planning, working memory, cognitive shifting, and inhibition - a set of mental skills that are important during continuous actions involving IPS. ${ }^{27}$ During IPS, one needs to engage the aforementioned cortical regions to retain information about the goal/task requirements and the spatial relationships between oneself and the partner, plan one's actions, shift one's attention to critical components of the actions, and inhibit irrelevant movements to match up with the partner's actions. These cortical regions not only interact with each other, but also with other cortical (e.g., occipital cortices) and subcortical regions (e.g., cerebellum is important for predictive control). ${ }^{28,29}$

\section{IPS difficulties in children with ASD}

Children and adolescents with ASD show reduced IPS during joint pendulum swaying and synchronized marching and clapping. 5,6 They also showed atypical movement characteristics during IPS (i.e., reduced temporal accuracy and altered movement speed) suggesting impaired social perception and solo coordination of actions. ${ }^{5,12}$ More specifically, impairments in goal 
understanding, social monitoring, executive functioning, as well as poor anticipatory and reactive control of movements in children with ASD might contribute to their difficulties in engaging in imitation and IPS with others. ${ }^{30-33}$

\section{Atypical cortical activation during IPS in children with ASD}

Besides the behavioral findings, children with ASD also show atypical cortical activation during imitation/IPS and its component behaviors (i.e., action observation and execution). Multiple fMRI studies have reported atypical activation over frontoparietal and superior temporal regions in children with ASD when imitating finger movements and hand gestures. ${ }^{34,35}$ While some electroencephalography research fails to duplicate the findings of atypical activation ${ }^{36}$, a thorough meta-analysis of imitation task data from fMRI studies found that individuals with ASD had increased IPL activation and altered activation over the occipital, dorsolateral prefrontal, and cingulate cortices, as well as the insula, compared to control participants. ${ }^{14}$

\section{functional Near-Infrared Spectroscopy (fNIRS)}

Although fMRI studies have identified possible neurobiomarkers of atypical imitation in individuals with ASD, the tasks were limited to simple finger and hand movements due to the constraints of the fMRI scanning bore. Functional near-infrared spectroscopy (fNIRS) is a noninvasive neuroimaging technique that constrains the subject only by a cap, allows upright face to face interactions, and is robust against movement artifacts ${ }^{37}$ making it a favorable approach to implement in children with ASD. Using fNIRS, our research group has reported atypical cortical activation, i.e., reduced IFG and STS activation, and increased IPL activation in children with ASD during a face to face reaching synchrony task. ${ }^{13}$ 


\section{Aims and hypotheses}

In the present study, we extended our past research to a whole-body, sway synchrony task, expanded the fNIRS probe set to better cover the prefrontal cortices as well, used motion tracking to objectively assess body movement characteristics, and manipulated social information during the sway synchrony task. We hypothesize that the children with ASD will have atypical IPS and sway amplitude compared to their TD peers. They will also have atypical frontoparietal and temporal activation during sway synchrony compared to TD peers. Moreover, we hypothesize that the sway characteristics and cortical activation of children with ASD will correlate with each other and with their social communication performance.

\section{Results}

\section{Movement characteristics during IPS}

Movement characteristics differed between children with and without ASD in the Social (Face and Touch) but not Solo conditions. Specifically, both groups showed similar sway amplitude in the Solo $(p>0.05)$ but children with ASD showed reduced sway amplitude in the Face and Touch conditions compared to the TD children ( $p$ s $<0.05$, Figure 1a). For sway coherence with the tester, children with ASD showed significantly lower coherence compared to the TD children in both Face and Touch conditions ( $p$ s $<0.01$, Figure 1b) indicating poor IPS in children with ASD.

\section{Figure 1.}




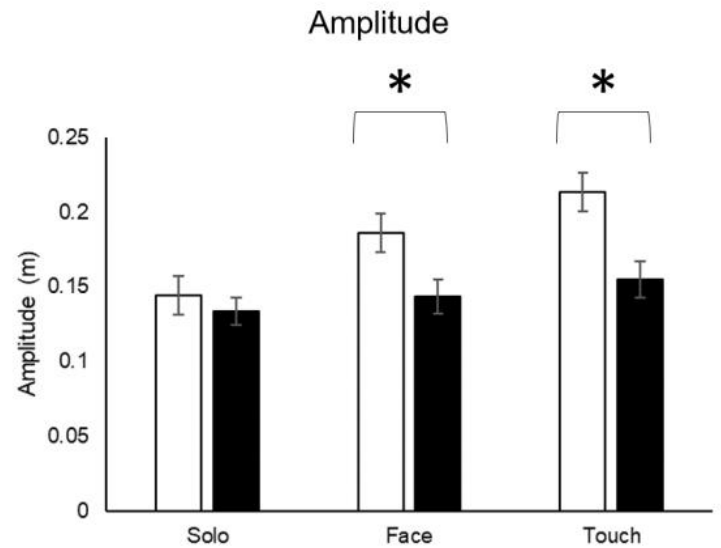

(a)

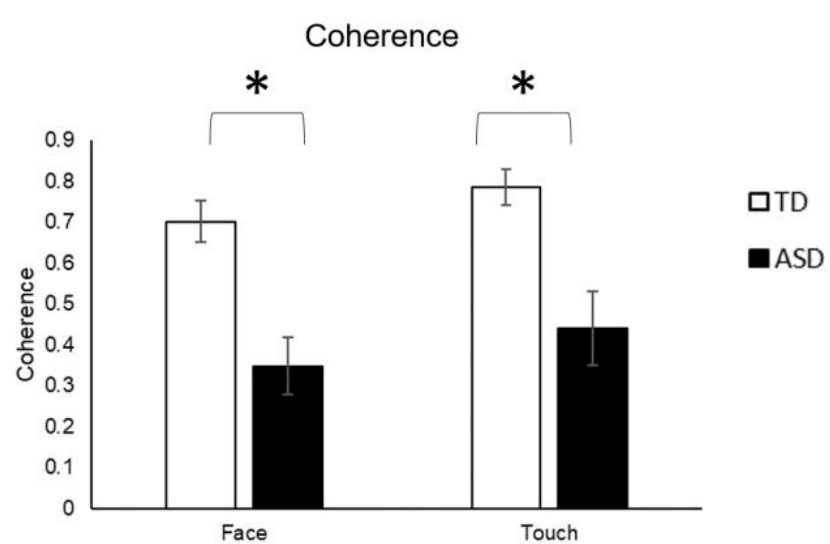

(b)

\section{Cortical activation during IPS}

The Group $\times$ Condition $\times$ Hemisphere $\times$ Region four-way repeated ANOVA revealed a significant main effect of Hemisphere $(F(1.0,115.0)=6.034, p=0.016)$, a 2-way interaction of Condition $\times$ Region $(\mathrm{F}(8.3,955.1)=2.5, p<0.05)$, and a 3-way interaction of Group $\times$ Condition $\times$ Region $(\mathrm{F}(8.3,955.1)=1.9, p=0.05)$. The Group $\times$ Condition $\times$ Region interaction did not covary with the sway amplitude, therefore, post-hoc analyses were done to further explore this interaction. The visual representation of averaged $\mathrm{HbO}_{2}$ concentration during all four conditions in both groups is shown in Figure 2. The means and standard errors ( $\mathrm{SE}$ ) of $\mathrm{HbO}_{2}$ concentrations are presented in Supplementary Table S1 and the results of post hoc analyses are presented in Supplementary Table S2 online. 


\section{Figure 2.}

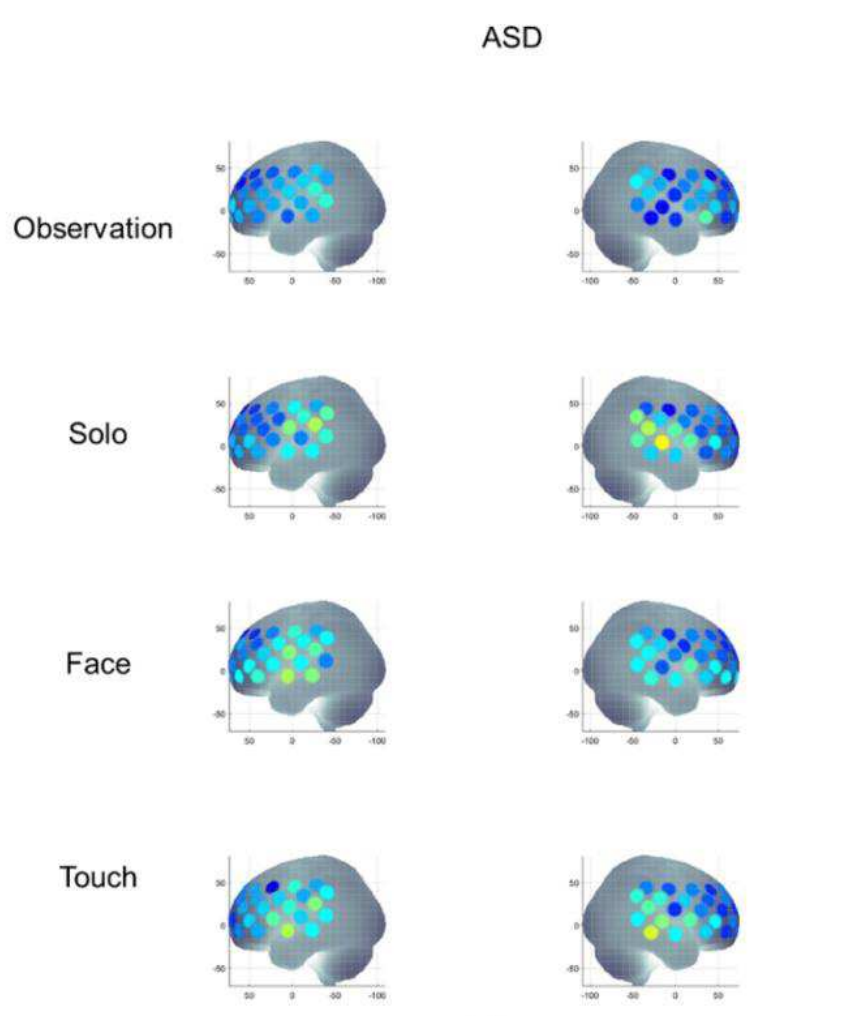

(a)
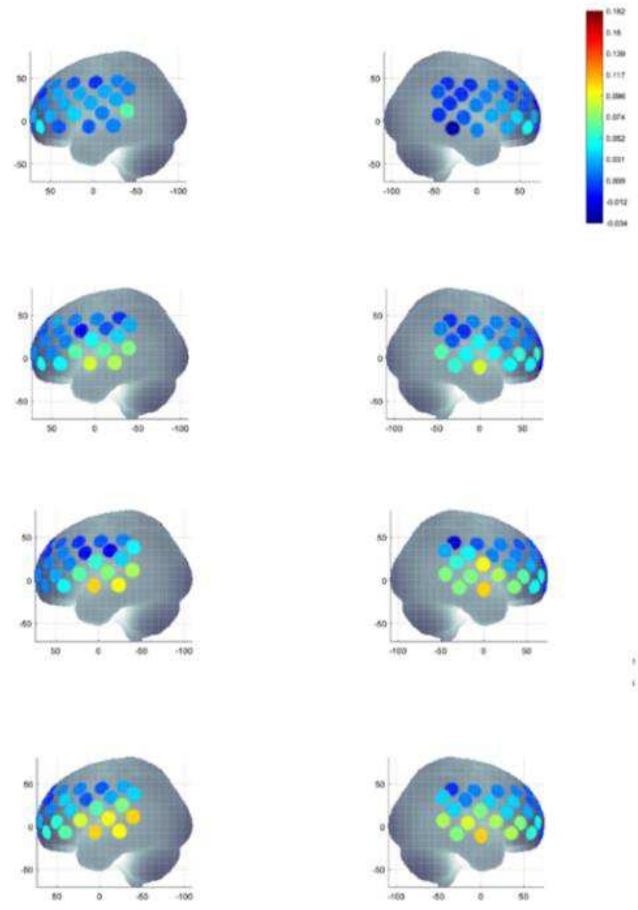

(b)

\section{Group differences}

During Observation and Solo conditions, children with ASD showed greater IPL activation than TD children $(p s<0.01$, Figures 3a and 3b). During the Face condition, children with ASD showed lower STS activation than the TD children $(p<0.01$, Figure 3c). Similarly, in the Touch condition, children with ASD had lower IFG and STS activation than the TD children $(p s<0.01$,

\section{Figure 3d).}

\section{Figure 3.}




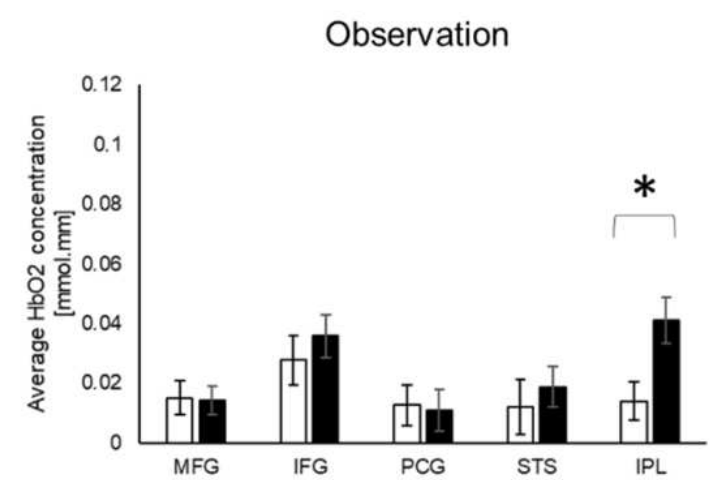

(a)

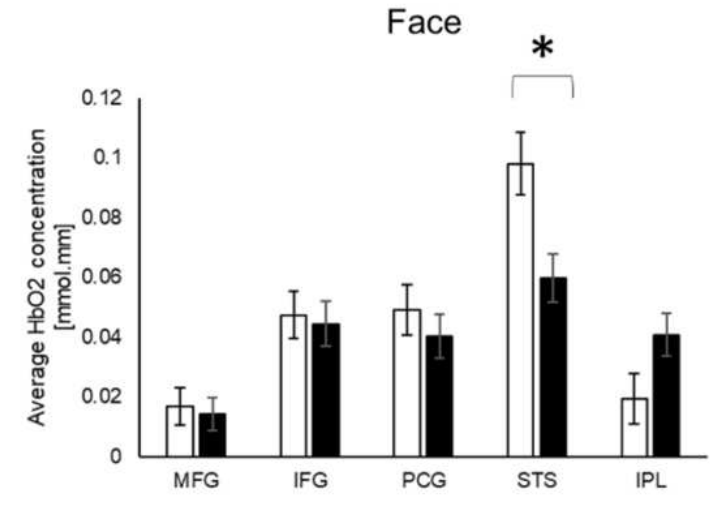

(c)

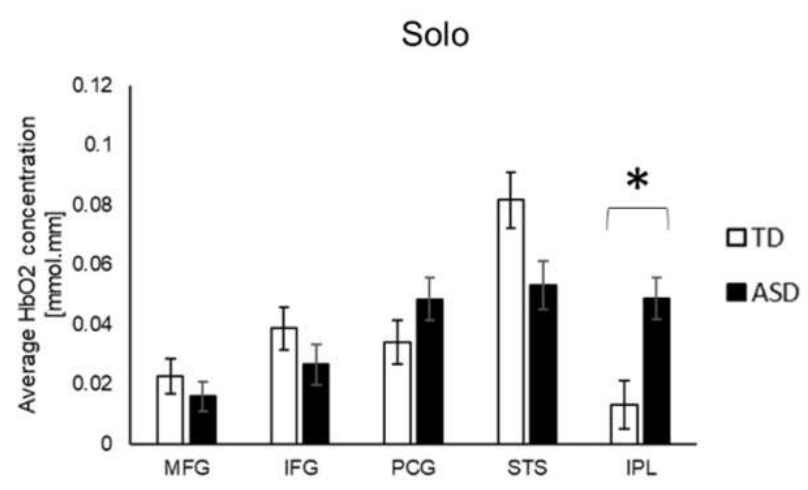

(b)

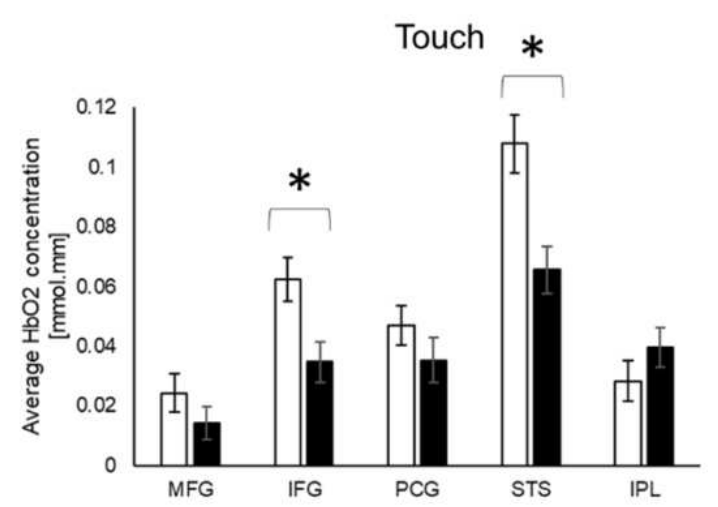

(d)

\section{Condition-related differences}

Both groups showed greater PCG and STS activation during the movement conditions (Solo, Face, and Touch) compared to the Observation condition ( $p s<0.05$, Figures 4a and 4b). In the movement conditions, the TD children scaled up their IFG and STS activation when more social information was available (IFG: Touch > Face and S, $p$ s < 0.05; PCG: Touch > Solo, $p$ s < 0.05, Figure 4a). However, children with ASD had no significant differences between movement conditions $($ Solo $\approx$ Face $\approx$ Touch, $p$ s $>0.05$, Figure $4 \mathbf{b})$.

\section{Figure 4.}




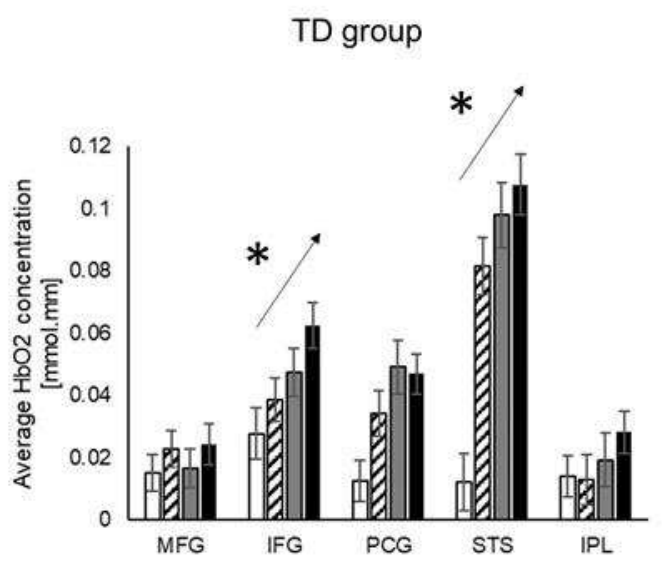

(a)

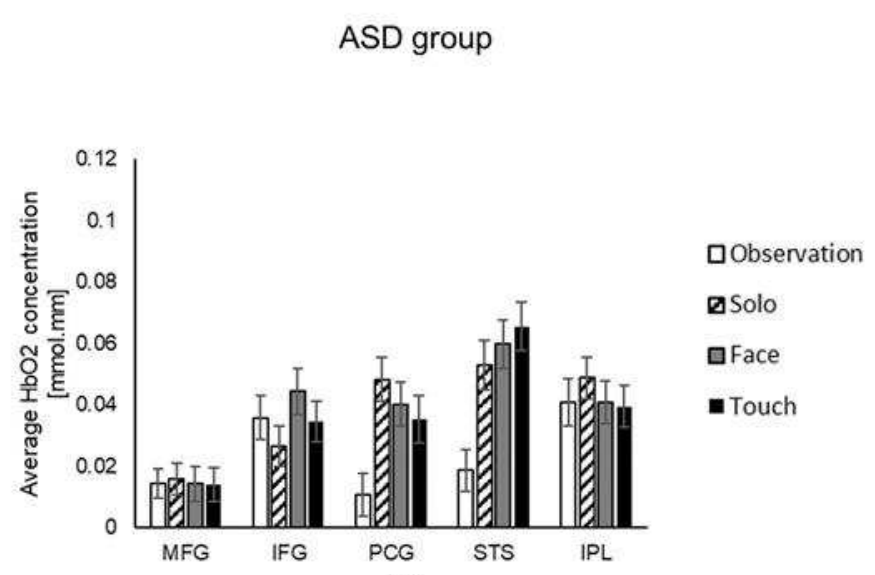

(b)

\section{Correlation between IPS behaviors and cortical activation}

In the TD children, greater sway amplitude was associated with greater right PCG and IPL activation during the Face condition (right PCG: $r=0.46$, right IPL: $r=0.41, p s<0.001$ ) whereas in the children with ASD, greater sway amplitude was associated with greater left STS and right PCG activation during the Touch condition (left STS: $r=0.48$, right PCG: $r=0.42, p s<0.001$ ). In the TD children, greater sway coherence was associated with greater right PCG activation during the Face condition $(\mathrm{r}=0.45, p<0.001)$; however, there was no significant correlation between coherence and cortical activation in the children with ASD (Table 1).

[Insert Table 1 here]

\section{Correlation between social communication questionnaires and cortical activation}

Only two correlations were noted in the TD group: Greater VABS communication scores were associated with greater left MFG activation during the Face condition $(\mathrm{r}=0.37, p<0.001)$ whereas greater VABS socialization scores were associated with lower right IFG activation during the Touch condition $(r=-0.43, p<0.001)$. In the ASD group, better VABS communication performance was associated with greater right PCG activation during the Face condition $(\mathrm{r}=0.41$, 
$p<0.001$ ), and greater left STS, left IPL, and right PCG activation during the Touch condition (rs $>0.45, p s<0.001)$. Furthermore, better VABS socialization performance was associated with greater right PCG activation ( $\mathrm{r}=0.45, p<0.001)$, better SRS performance (i.e., lower SRS scores) was associated with greater left STS and right PCG activation (rs $<-0.35, p<0.001$ ), and greater ICS score was associated with greater left IFG activation during the Touch condition $(r=0.44, p$ $<0.001$, Table 2).

[Insert Table 2 here]

\section{Discussion}

Children with ASD have social and perceptuo-motor difficulties that affect their abilities to engage in IPS. ${ }^{6}$ Previous fMRI and fNIRS studies have reported atypical cortical activation associated with imitation and IPS difficulties in adults and children with ASD. ${ }^{13,14}$ However, the studies were limited to finger movements or seated reaching tasks without involving whole-body movements. The present study extended our past work studying reaching-related IPS to a whole-body sway synchrony task and investigated how different levels of social information might affect the IPS behaviors and cortical activation in children with and without ASD.

Consistent with past studies, children with ASD had reduced IPS and sway amplitude compared to the TD children. In terms of cortical activation, children with ASD showed greater IPL activation during the Observation and Solo conditions, reduced STS activation during the Face condition, and reduced IFG and STS activation during the Touch condition. When more social information was provided (social-visual and/or social-visual and tactile), children with ASD failed to scale up their IFG and STS activation unlike their TD peers. Cortical activation in children with 
ASD correlated with their movement characteristics during the sway synchrony task as well as their social communication performance.

Children with ASD showed reduced IPS and insufficient sway amplitude compared to the TD children. The group differences in sway amplitude were only seen in the IPS conditions (Face and Touch) but not the Solo condition, indicating differences during socially embedded actions. Similar IPS difficulties have been reported in children with ASD during synchronized pendulum swaying, ${ }^{5}$ marching and clapping, ${ }^{6}$ and virtual tightrope walking tasks. ${ }^{54}$ During the aforementioned tasks, children with ASD showed lower temporal accuracy indicating lower coordination and greater variability. ${ }^{5,54}$ The IPS difficulties of children with ASD might be rooted in their poor multi-limb and visuo-motor coordination leading and a greater challenge during social synchrony ${ }^{54,55}$ Besides the motor components, children with ASD have social perceptual deficits (i.e., reduced social gaze, or poor perception of other's actions) which could also undermine their ability to produce synchronized actions. ${ }^{56}$ Together, these sensory-motor and social-perceptual difficulties may contribute to poor IPS performance in children with ASD.

We found greater IPL activation in children with ASD during Observation and Solo conditions compared to the TD children. Similar findings of hyperactivation over the IPL region in children with ASD have been reported in previous fMRI and fNIRS studies involving simple finger and hand movement imitation/IPS. ${ }^{13,14}$ The IPL region is part of the default mode network (DMN) $)^{57}$, a network that is more active during internally directed processing (i.e., mind wandering and selfreferencing), but is deactivated during externally directed processing (i.e., performing cognitively demanding tasks and goal-directed movements such as IPS). ${ }^{58} \mathrm{DMN}$ is important in a wide range of emotions and social functions and share similar cortical substrates including regions important for imitation. ${ }^{59,60}$ An fMRI study found more DMN deactivation when being imitated, compared 
to when individuals were imitating other's movements, suggesting that DMN is more active when trying to match up to another individual's actions. ${ }^{60}$ Consistent with the previous finding, the TD children showed more deactivation during Observation and Solo conditions and less deactivation/more activation over the IPL regions during Face and Touch conditions. Children with ASD in general did not show deactivation of the IPL region during all conditions and had much greater overall IPL activation compared to TD children. It is possible that children with ASD were less focused on the externally-directed goals of solo/social synchrony and had difficulties deactivating the IPL region. Children with ASD are known to have problems with deactivation during other cognitive tasks requiring visual inspection of complex patterns as well as working memory tasks. ${ }^{61,62}$

Consistent with the previous fMRI and fNIRS findings ${ }^{13,14}$, we found hypoactivation in IFG and STS in children with ASD during IPS. The IFG and STS activation was associated with the social communication and IPS performance in children with ASD. IFG is important for goal/intention understanding during action execution and imitation. ${ }^{19,28}$ While observing object manipulation, the IFG was more active when the participants were told to focus on the goal but not the actions associated with the task. ${ }^{63}$ Children with ASD have difficulties understanding goals or intentions underlying others' actions ${ }^{33}$, and the difficulties have been linked to lower IFG activation during action observation. ${ }^{64}$ During the sway synchrony task in this study, children with ASD might have focused more on their individual movements instead of the shared goal (i.e., moving in synchrony with the adult), and therefore showed reduced IFG activation during IPS.

On the other hand, the STS region is important for establishing visuo-motor correspondence, a process that compares the observed action with the planned movement, which is much needed for imitation. ${ }^{24,65}$ STS is more active during imitation compared to action execution and observation 
across different tasks, including actions involving simple finger movements ${ }^{25}$, and pantomimed actions on objects. ${ }^{24}$ Children with ASD have decreased STS activation when observing biological motions and during action imitation. ${ }^{66,67}$ Similarly, we found reduced STS activation in children with ASD during sway synchrony, suggesting that they have difficulties matching the observed actions with their own movement repertoire. Through this study, we extend our previous neurobiomarkers during reaching synchrony ${ }^{13}$ to whole-body sway synchrony and reconfirmed that the ASD-related neurobiomarkers are associated with IPS impairments regardless of movements performed (i.e., reaching or whole-body sway).

We found a clear increase in cortical activation when more social perceptual information (i.e., social visual and/or social tactile information) was available to the TD children. Similar results were reported in previous fMRI studies, with increased frontoparietal activation during multisensory processing). ${ }^{21}$ The additional social visual (Face condition) and social visual plus social tactile information (Touch condition) might increase the information reaching the frontoparietal regions, leading to scaling up of cortical activation. However, children with ASD might have difficulties perceiving/processing multiple social inputs (tactile and visual) as they failed to scale up their cortical activation when more social information was available. Children with ASD have difficulties integrating multiple sensory (visual and tactile) and social perceptual inputs. ${ }^{68,69}$ These difficulties in perceiving task-relevant information could affect their ability to sway and engage in IPS with others.

To our knowledge, this is the first study to investigate how different levels of social information affect whole-body IPS and cortical activation patterns in children with and without ASD. Although we identified the neurobiomarkers in children with ASD, there were some limitations to our study, including a relatively small sample size and the lack of eye-tracking measures to study social gaze. 
We did not include any cognitive measures, but we have correlated social communication functioning to cortical activation measures in both groups.

Using motion tracking and fNIRS systems, we identified atypical movement characteristics and associated cortical activation in children with ASD during a whole-body, sway synchrony task. Compared to the TD children, children with ASD showed hyperactivation in the IPL region during action observation and execution and showed reduced IFG and STS activation during socialembedded synchronous sway. Moreover, they failed to scale up IFG and STS activation when more social information was available. The ASD-related atypical cortical activation patterns associated with IPS noted in the present study could be addressed through synchrony-based interventions. ${ }^{70,71}$ In fact, we plan to use the fNIRS-based neurobiomarkers identified in the present study as objective markers of treatment response following such interventions.

\section{Methods}

\section{Participants}

Thirty school-aged children with and without ASD participated in this study (mean age \pm SE: ASD: $12.7 \pm 0.8$ years, 12 males and 3 females; TD: $12.5 \pm 0.8$ years, 10 males and 5 females; $p$ s $>$ 0.05). Participants were recruited through online announcements, phone calls and fliers distributed to local schools, ASD service and advocacy groups, as well as Simons Powering Autism Research (SPARK) participant research match service. SPARK informs their database of families about research studies in the nearby area (https://www.sfari.org/resource/spark/). Before participation, we interviewed potential participants to obtain their demographic information including age, sex, ethnicity, as well as to confirm their eligibility for participation (Table 3). Children with ASD were included if they held a professionally confirmed ASD diagnosis 
supported by a school record (e.g., school psychologist record confirming an ASD diagnosis) and an Individualized Education Plan (IEP) for ASD-related services or a medical or neuropsychological record from a psychiatrist or clinical psychologist using the Autism Diagnostic Observation Schedule (ADOS) and/or Autism Diagnostic Interview-Revised (ADI-R) measures. In addition, we used the Social Communication Questionnaire (SCQ) ${ }^{38}$ to screen for ASD symptoms. All children with ASD met criteria for a social communication delay (>15 points) confirming the presence of a social communication difficulties, whereas all TD children scored below the cut-off score. Additionally, the two groups were matched in age and sex. Children with ASD were excluded if they had any behavioral and sensory issues that prevented them from wearing the fNIRS cap and completing the test activities. TD children were excluded if they: a) had any neurological or developmental disorder/delays or b) had a family history of ASD.

Parents of all participating children were asked to complete the Vineland Adaptive Behavioral Scales-2 ${ }^{\text {nd }}$ edition $(\mathrm{VABS})^{39}$, Social Responsive Scale questionnaire $(\mathrm{SRS})^{40}$, and the Interpersonal Competence Scale (ICS) ${ }^{41}$ to assess the child's social communication performance (Table 3). Compared to the TD children, children with ASD had significantly lower VABS, SRS, and ICS scores ( $\underline{s}<0.05$, Table 3). Lastly, the Coren's handedness survey was used to determine children's handedness (Table 3) ${ }^{42}$ Written informed consent was obtained from parents and written/verbal assent was received from participants prior to study participation. All study procedures were carried out in accordance with the Declaration of Helsinki. All consent and assent forms as well as all study procedures were approved by the University of Delaware Institutional Review Board (UD IRB, Study Approval \#: 930721-12). Prior to study participation, written informed consent was obtained from parents who gave approval on behalf of their children as their legal guardians and written and verbal assent was obtained from the 
children. Written parental and experimenter permission/consent to use their pictures for this publication has been taken.

[Insert Table 3 here]

\section{Experimental procedures}

During the experiment, the children were asked to stand either facing a laptop screen or an adult tester. An fNIRS cap embedded with a $3 \times 11$ probe set was placed on each child's head. Each child completed four conditions: Observation, Solo, Face, and Touch (Figure 5a). During the Observation condition, the child observed an adult tester sway side to side. During the Solo condition, the child swayed side to side in synchrony with an oscillating bar shown on the laptop screen. During the Face condition, the child swayed face to face with an adult tester. Lastly, during the Touch condition, the child swayed face to face with an adult tester with their fingertips touching each other. The swaying speed of the tester and the oscillating bar were controlled by a rhythmic beat audible to the tester only using a wireless earphone, therefore the child had to synchronize their sway with the tester using social-visual and/or social-tactile information. The speed of the bar/tester's motions changed within the trial, either moved faster from 40-50 bpm, or slower from 40-30 bpm. The children completed a total of 16 trials (4 trials per condition) that were randomized across the entire session (Figure 5b). Each trial included a 10-second pre-stimulation, 25-second stimulation, and a 25-second post-stimulation period. The pre- and post-stimulation periods were included to account for baseline drifts in the fNIRS signal and to allow the hemodynamic response to return to baseline before starting the next trial. During baseline periods, the children were asked to stand still and focus on a crosshair on the front wall.

\section{Figure 5.}




\section{Experimental Procedures}
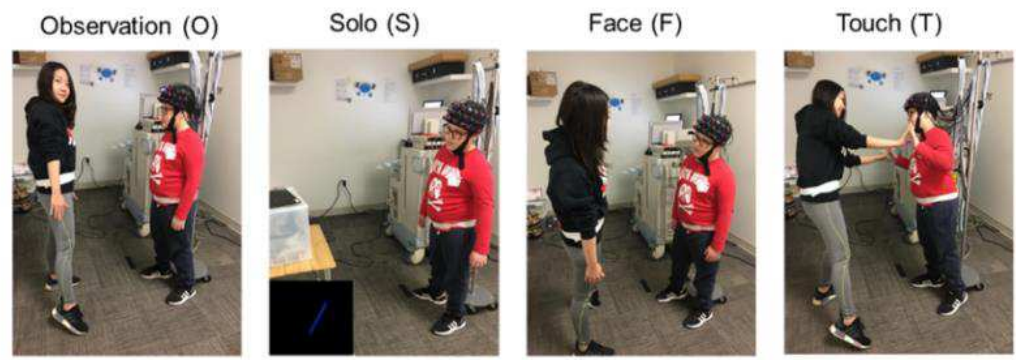

(a)

Trial Sequence in E-Prime

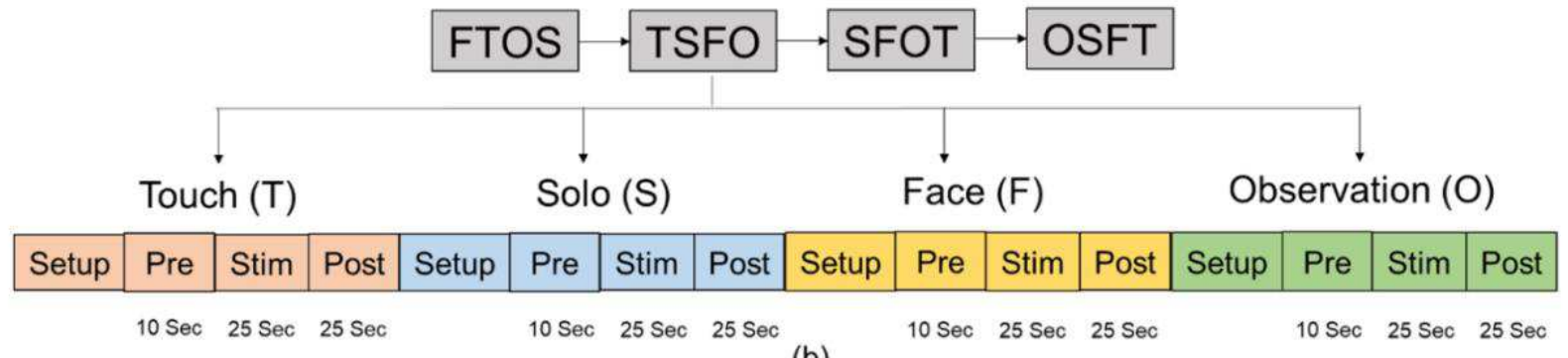

(b)

\section{Data collection}

We used a 52-channel Hitachi fNIRS system (ETG-4000, Hitachi Medical Systems, Inc., sampling rate: $10 \mathrm{~Hz}$ ) to record the hemodynamic changes over the regions of interest (ROIs). A $3 \times 11$ probe set consisting of 17 infrared emitters and 16 receivers was positioned over bilateral frontoparietal and temporal regions. The middle column of the probe set was aligned with the child's nasion while the bottom row was aligned with the child's eyebrow (See Supplementary Fig. S1a online). Each adjacent pair of probes are located $3 \mathrm{~cm}$ apart on the cap and act as an emitter and a receiver of two wavelengths of infrared light (695 and $830 \mathrm{~nm}$ ). Infrared light passes through the skull creating a banana-shaped arc and reaches the cortical area approximately below the midpoint of the two probes forming a channel. The attenuation of infrared light was used to calculate the changes in concentrations of oxygenated $\left(\mathrm{HbO}_{2}\right)$ and deoxygenated hemoglobin chromophores $(\mathrm{HHb})$ per channel using the Modified Beer-Lambert Law. ${ }^{37}$ An increase in the 
concentration of $\mathrm{HbO}_{2}$ and a decrease in $\mathrm{HHb}$ concentration were expected when a certain region underneath a channel was active. ${ }^{37}$ Additionally, inertial measurement units (IMUs, Xsens, Inc.) were placed on the mid-point of the second sacral vertebrae (i.e., body's center of mass) for the child and the tester to record body displacements during swaying (Sampling rate: $100 \mathrm{~Hz}$; see Supplementary Fig. S1c online). The Hitachi fNIRS system and the IMU systems were synchronized and triggered by E-Prime presentation software (version 2.0). The entire session was videotaped using a camcorder that was also synchronized with the fNIRS system.

\section{Spatial registration approach}

We used the ETG-4000 3D positioning unit, a reference coordinate system, to conduct 3D registration. During registration, we recorded the 3D locations of the standard cranial landmarks (i.e., nasion, inion, tragus points of the ears, and the Cz position of the International 10-20 system) and each of the probes. The 3D coordinates of all participating children were run through MATLAB codes developed by our collaborator/co-author Dr. Tsuzuki ${ }^{43}$ to estimate and label the channel locations using the LONI Probabilistic Brain Atlas (LPBA) ${ }^{44}$ For further details, readers may refer to our earlier publications. ${ }^{13,45,46} \mathrm{~A}$ channel was included if more than $60 \%$ of a channel (i.e., each channel was as the centroid of sphere) was within a given ROI and was excluded if it was not. A channel was also excluded if its homologue belonged to another ROI (e.g., the homologous channels 12 and 20 were excluded because channel 12 was assigned to IPL region while channel 20 was assigned to the PCG region). Based on these rules, we assigned 32 out of the 52 channels to five ROIs in each hemisphere (See Supplementary Fig. S1b and Table S3 online): i) MFG (left: channels 7, 8, 17, 18, 28, 38; right: channels 3, 4, 14, 15, 25, 36); ii) IFG (left: channels 29, 39, 50; right: channels 24, 35, 45); iii) PCG (left: channels 9, 30; right: channels 
2, 23); iv) STS (left: channels 42, 51, 52; right: channels 32, 43, 44); and v) IPL (left: channels 10 , 21; right: channels 1,11$)$.

\section{Data processing}

Data on displacement of the body's center of mass were obtained from the child and the adult and were later analyzed using custom MATLAB codes. First, we low-pass filtered the displacement data using a $4^{\text {th }}$ order, zero-lag Butterworth filter using a cut-off frequency of $10 \mathrm{~Hz}$. Then, we extracted the amplitudes in mm for each child. To calculate IPS, we used Cross-Spectral Analysis $(\mathrm{CSA})^{47}$ to determine the level of coherence between the child and the tester. Coherence is a measure of the degree of correlation between the signals within a certain frequency and it ranges from 0 to 1 (i.e., 0 presents no synchrony and 1 presents perfect synchrony). The CSA approach had been used in previous studies to analyze correlations between rhythmic sway data between two individuals. ${ }^{48}$

To analyze the fNIRS data, we incorporated open-source software - Hitachi POTATo ${ }^{49}$ and Homer- $2^{50}$ - within custom MATLAB codes. Specifically, we band-pass filtered the data between 0.01 and $0.5 \mathrm{~Hz}$ to remove high- and low-frequency noise, used the wavelet method to remove movement artifacts, used General Linear Modeling (GLM) to estimate the hemodynamic response function, and corrected for baseline drifts by subtracting the linear trend between the pre- and poststimulation baselines from values in the stimulation period. ${ }^{49,50}$ Lastly, we averaged the $\mathrm{HbO}_{2}$ and $\mathrm{HHb}$ values over each stimulation period for each trial. $\mathrm{The}_{\mathrm{HbO}}$ data has a greater range and signal to noise ratio compared to the $\mathrm{HHb}$ data and is more often reported in the literature. ${ }^{51}$ The data were plotted and saved at each step for visual screening. For additional details, readers may refer to our earlier publications. . $^{13,45,46}$ 


\section{Data exclusion}

Cortical activation and movement kinematic data were excluded based on visual screening of video and MATLAB figures. A trained student researcher scored the session videos to exclude trials with significant behavioral errors (e.g., child did not follow task instructions or spoke to the tester during the stimulation period). This criterion resulted in data exclusion of 5\% from the ASD group and $1 \%$ from the TD group. In addition, we screened the MATLAB figures of cortical activation data at each step to exclude channels with no signals (i.e., appeared as flat lines due to bad probe-scalp contact). $12 \%$ of data from the ASD and $5 \%$ of data from the TD groups were removed based on channel checking. In total, $15 \%$ data from the ASD group and $6 \%$ data from the TD group and were excluded and $85-94 \%$ of data were retained in our analysis.

\section{Statistical analyses}

For sway amplitude and coherence data from the IMU system, t-tests were used to compare group/condition-related differences. For the cortical activation data, we averaged data across channels within the same ROI based on our spatial registration output (See Supplementary Fig. S1b online) to avoid multiple comparisons. We determined levels of activation for 10 ROIs, including the MFG, IFG, PCG, STS, and IPL regions for both left and right hemispheres. Using IBM SPSS (SPSS, Inc.), a repeated-measures ANOVA was conducted using within-subject factors of condition (Observation, Solo, Face, Touch), hemisphere (left, right), and ROI (MFG, IFG, PCG, STS, IPL) and a between-subjects factor of group (TD, ASD) for average $\mathrm{HbO}_{2}$ values. Since we found group differences in movement amplitude, we added that as a covariate to control for cortical activation associated with differences in movement characteristics. Greenhouse-Geisser corrections were applied when our data violated the sphericity assumption based on Mauchly's test of sphericity. Differences in social communication skills are associated with the ASD 
diagnosis and ideally should not be controlled by treating them as covariates, ${ }^{52}$ therefore we used Pearson's correlations to investigate the relationships between cortical activation, movement characteristics, and social communication performance assessed by three measures - VABS, SRS, and ICS.

To account for multiple comparisons (post hoc t-tests following ANOVA runs and correlational analyses), we used the False Discovery Rate (FDR) method to adjust the cut-off for statistical significance. ${ }^{53}$ Specifically, the Benjamin-Hochberg method was used wherein unadjusted $p$ values were rank ordered from low to high. Statistical significance was declared if the unadjusted $p$-value was less than the $p$-value threshold. $p$-value threshold was determined by multiplying 0.05 with the ratio of the unadjusted $p$-value rank to the total number of comparisons ( $p$-threshold for $\mathrm{i}^{\text {th }}$ comparison $=0.05 \times \mathrm{i} / \mathrm{n}$; where $\mathrm{n}=$ total number of comparisons).

\section{Data availability statement}

The datasets generated during and/or analysed during the current study are available from the corresponding author on reasonable request.

\section{References}

1. Baio, J. et al. Prevalence of autism spectrum disorder among children aged 8 years - autism and developmental disabilities monitoring network, 11 sites, United States, 2014. MMWR Surveill. Summ. 67(6), 1-23 (2018).

2. American Psychiatric Association. Diagnostic and statistical manual of mental disorders. $5^{\text {th }}$ ed. Washington, D.C (American Psychiatric Publishing, 2013). 
3. Bhat, A.N., Landa, R.J., \& Galloway, J.C. Current perspectives on motor functioning in infants, children, and adults with autism spectrum disorders. Phys. Ther. 91(7), 1116-1129 (2011).

4. Edwards, L. A. A meta-analysis of imitation abilities in individuals with autism spectrum disorders. Autism Res. 7(3), 363-380 (2014).

5. Fitzpatrick, P. et al. Impairments of social motor synchrony evident in autism spectrum disorder. Front Psychol. 7, 1323 (2016).

6. Kaur, M., Srinivasan, S.M., \& Bhat, A.N. Comparing motor performance, praxis, coordination, and interpersonal synchrony between children with and without Autism Spectrum Disorder (ASD). Res. Dev. Disabil. 72 ,79-95 (2018).

7. Iverson, J.M. Early motor and communicative development in infants with an older sibling with autism spectrum disorder. J. Speech Lang. Hear Res. 61(11), 2673-2684 (2018).

8. Cirelli, L.K., Trehub, S.E., \& Trainor, L.J. Rhythm and melody as social signals for infants. Ann NY Acad. Sci. 10.1111/nyas.13580 (2018).

9. Bureau, J.F. et al. Making children laugh: parent-child dyadic synchrony and preschool attachment. Infant Ment. Health J. 35(5), 482-494 (2014).

10. Tunçgenç, B., \& Cohen, E. Movement synchrony forges social bonds across group divides. Front. Psychol. 7, 782 (2016).

11. Mogan, R., Fischer, R., \& Bulbulia, J.A. To be in synchrony or not? A meta-analysis of synchrony's effects on behavior, perception, cognition and affect. J. Exp. Soc. Psychol. 72, 1320 (2017). 
12. Fitzpatrick, P. et al. Evaluating the importance of social motor synchronization and motor skill for understanding autism. Autism Res. 10(10), 1687-1699 (2017).

13. Su, W.C. et al. Differences in cortical activation patterns during action observation, action execution, and interpersonal synchrony between children with or without autism spectrum disorder (ASD): An fNIRS pilot study. PLoS One. 15(10), e0240301 (2020).

14. Yang, J., \& Hofmann, J. Action observation and imitation in autism spectrum disorders: an ALE meta-analysis of fMRI studies. Brain Imaging Behav. 10(4), 960-969 (2016).

15. Cheng, M., Kato, M., \& Tseng, C.H. Gender and autistic traits modulate implicit motor synchrony. PLoS One. 12(9), e0184083 (2017).

16. Josef, L., Goldstein, P., Mayseless, N., Ayalon, L., \& Shamay-Tsoory, S.G. The oxytocinergic system mediates synchronized interpersonal movement during dance. Sci. Rep. 9(1), 1894 (2019).

17. Vesper, C. et al. Joint action: mental representations, shared information and general mechanisms for coordinating with Others. Front. Psychol. 7, 2039 (2017).

18. Iacoboni, M. Neural mechanisms of imitation. Curr. Opin. Neurobiol. 15(6), 632-637 (2005).

19. Koski, L. et al. Modulation of motor and premotor activity during imitation of target-directed actions. Cereb. Cortex. 12(8), 847-855 (2002).

20. Johnson-Frey, S.H. et al. Actions or hand-object interactions? Human inferior frontal cortex and action observation. Neuron. 39(6), 1053-1058 (2003). 
21. Limanowski, J., \& Blankenburg, F. Fronto-parietal brain responses to visuotactile congruence in an anatomical reference frame. Front. Hum. Neurosci. 12, 84 (2018).

22. Fontana, A.P. et al. Role of the parietal cortex in predicting incoming actions. NeuroImage. 59(1), 556-564 (2012).

23. Ocampo, B., Kritikos, A., \& Cunnington, R. How frontoparietal brain regions mediate imitative and complementary actions: an FMRI study. PLoS One. 6(10), e26945 (2011).

24. Molenberghs, P., Brander, C., Mattingley, J.B., \& Cunnington, R. The role of the superior temporal sulcus and the mirror neuron system in imitation. Hum. Brain Mapp. 31(9), 13161326 (2010).

25. Gatti, R. et al. The effect of action observation/execution on mirror neuron system recruitment: an fMRI study in healthy individuals. Brain Imaging Behav. 11(2), 565-576 (2017).

26. Javed, K., Reddy, V., \& Lui, F. Neuroanatomy, Cerebral Cortex. StatPearls (StatPearls Publishing, 2020).

27. Del Casale, A. et al. Executive functions in obsessive-compulsive disorder: An activation likelihood estimate meta-analysis of fMRI studies. World J. Biol. Psychiatry 17(5), 378-393 (2016).

28. Iacoboni, M. Neurobiology of imitation. Curr. Opin. Neurobiol. 19(6), 661-665 (2009).

29. Menz, M.M., McNamara, A., Klemen, J., \& Binkofski, F. Dissociating networks of imitation. Hum. Brain Mapp. 30(10), 3339-3350 (2009). 
30. Chen, L.C. et al. Postural control and interceptive skills in children with autism spectrum disorder. Phys. Ther. 99(9), 1231-1241 (2019).

31. Chita-Tegmark, M. Social attention in ASD: A review and meta-analysis of eye-tracking studies. Res. Dev. Disabil. 48, 79-93 (2016).

32. Demetriou, E.A. et al. Autism spectrum disorders: a meta-analysis of executive function. Mol. Psychiatry 23(5), 1198-1204 (2018).

33. Somogyi, E., Király, I., Gergely, G., \& Nadel, J. Understanding goals and intentions in lowfunctioning autism. Res. Dev. Disabil. 34(11), 3822-3832 (2013).

34. Wadsworth, H.M. et al. The action imitation network and motor imitation in children and adolescents with autism. Neuroscience 343, 147-156 (2017).

35. Williams, J.H. et al. Neural mechanisms of imitation and 'mirror neuron' functioning in autistic spectrum disorder. Neuropsychologia 44(4), 610-621 (2006).

36. Fan, Y.T., Decety, J., Yang, C.Y., Liu, J.L., \& Cheng, Y. Unbroken mirror neurons in autism spectrum disorders. J. Child Psychol. Psychiatry 51(9), 981-988 (2010).

37. Lloyd-Fox, S., Blasi, A., \& Elwell, C.E. Illuminating the developing brain: the past, present and future of functional near infrared spectroscopy. Neurosci. Biobehav. Rev. 34(3), 269-284 (2010).

38. Rutter, M., Bailey, A., \& Lord, C. The social communication questionnaire: Manual. Los Angeles (Western Psychological Services, 2003). 
39. Sparrow, S.S., Cicchetti, D.V., \& Balla DA. Vineland adaptive behavior scales (2 ${ }^{\text {nd }}$ ed.). Antonio (NCS Pearson.San, 2005).

40. Constantino, J.N., Przybeck, T., Friesen, D., \& Todd, R.D. Reciprocal social behavior in children with and without pervasive developmental disorders. J. Dev. Behav. Pediatr. 21(1), 2-11 (2000).

41. Cairns, R.B., Leung, M.C., Gest, S.D., \& Cairns, B.D. A brief method for assessing social development: structure, reliability, stability, and developmental validity of the Interpersonal Competence Scale. Behav. Res. Ther. 33(6), 725-736 (1995).

42. Coren, S. The lateral preference inventory for measurement of handedness, footedness, eyedness, and earedness: norms for young adults. Bull. Psychon. Soc. 31, 1-3 (1993).

43. Tsuzuki, D., Cai, D.S., \& Dan, H. Stable and convenient spatial registration of stand-alone NIRS data through anchor-based probabilistic registration. Neurosci. Res. 72(2), 163-171 (2012).

44. Shattuck, D.W. et al. Construction of a 3D probabilistic atlas of human cortical structures. NeuroImage 39(3), 1064-1080 (2008).

45. Su, W.C. et al. Developmental differences in cortical activation during action observation, action execution and interpersonal synchrony: an fNIRS study. Front. Hum. Neurosci. 14, 57 (2020). 
46. Bhat, A.N. et al. Cortical activation during action observation, action execution, and interpersonal synchrony in adults: A functional near-infrared spectroscopy (fNIRS) study. Front. Hum. Neurosci. 11, 431 (2017).

47. Halliday, D.M. et al. A framework for the analysis of mixed time series/point process data Theory and application to the study of physiological tremor, single motor unit discharges, and electromyograms. Prog. Biophys. Mol. Biol. 64, 237-278 (1995).

48. Sofianidis, G., Elliott, M.T., Wing, A.M., \& Hatzitaki, V. Interaction between interpersonal and postural coordination during frequency scaled rhythmic sway: the role of dance expertise. Gait Posture 41(1), 209-216 (2015).

49. Sutoko, S. et al. Tutorial on platform for optical topography analysis tools. Neurophotonics 3(1), 010801 (2016).

50. Huppert, T.J., Diamond, S.G., Franceschini, M.A., \& Boas, D.A. HomER: a review of timeseries analysis methods for near-infrared spectroscopy of the brain. Appl. Opt. 48(10), D280D298 (2009).

51. Sato, H. et al. Intersubject variability of near-infrared spectroscopy signals during sensorimotor cortex activation. J. Biomed. Opt. 10(4), 44001 (2005).

52. Miller, G.A., \& Chapman, J.P. Misunderstanding analysis of covariance. J. Abnorm. Psychol. 110(1), 40-48 (2001).

53. Singh, A.K., \& Dan, I. Exploring the false discovery rate in multichannel NIRS. NeuroImage 33(2), 542-549 (2006). 
54. Xavier, J. et al. Interpersonal synchronization, motor coordination, and control are impaired during a dynamic imitation task in children with autism spectrum disorder. Front. Psychol. 9, 1467 (2018).

55. Nebel, M.B. et al. Intrinsic visual-motor synchrony correlates with social deficits in autism. Biol. Psychiatry. 79(8), 633-641 (2016).

56. Dawson, G. et al. Early social attention impairments in autism: social orienting, joint attention, and attention to distress. Dev. Psychol. 40(2), 271-283 (2004).

57. Grodd, W., Beckmann, C.F., \& Funktionelle, M.R.T. des gehirns im ruhezustand [Resting state functional MRI of the brain]. Der. Nervenarzt. 85(6), 690-700 (2014).

58. Greicius, M.D., Krasnow, B., Reiss, A.L., \& Menon, V. Functional connectivity in the resting brain: a network analysis of the default mode hypothesis. Proc. Natl. Acad. Sci. U. S. A. 100(1), 253-258 (2003).

59. Schilbach, L., Eickhoff, S.B., Rotarska-Jagiela, A., Fink, G.R., \& Vogeley, K. Minds at rest? Social cognition as the default mode of cognizing and its putative relationship to the "default system" of the brain. Conscious Cogn. 17(2), 457-467 (2008).

60. Guionnet, S. et al. Reciprocal imitation: toward a neural basis of social interaction. Cereb. Cortex 22(4), 971-978 (2012).

61. Spencer, M. D. et al. Failure to deactivate the default mode network indicates a possible endophenotype of autism. Mol. Autism 3(1), 15 (2012). 
62. Chien, H.Y., Gau, S.S., \& Isaac Tseng, W.Y. Deficient visuospatial working memory functions and neural correlates of the default-mode network in adolescents with autism spectrum disorder. Autism Res. 9(10), 1058-1072 (2016).

63. Nicholson, T., Roser, M., \& Bach, P. Understanding the goals of everyday instrumental actions is primarily linked to object, not motor-kinematic, information: evidence from fMRI. PLoS One 12(1), e0169700 (2017).

64. Pokorny, J.J. et al. The action observation system when observing hand actions in autism and typical development. Autism Res. 8(3), 284-296 (2015).

65. Pelphrey, K.A. et al. Brain activity evoked by the perception of human walking: controlling for meaningful coherent motion. J. Neurosci. 23(17), 6819-6825 (2003).

66. Freitag, C.M. et al. Perception of biological motion in autism spectrum disorders. Neuropsychologia 46(5), 1480-1494 (2008).

67. Jack, A., \& Morris, J.P. Neocerebellar contributions to social perception in adolescents with autism spectrum disorder. Dev. Cogn. Neurosci. 10, 77-92 (2014).

68. Greenfield, K., Ropar, D., Smith, A.D., Carey, M., \& Newport, R. Visuo-tactile integration in autism: atypical temporal binding may underlie greater reliance on proprioceptive information. Mol. Autism 6, 51 (2015).

69. Shah, P., \& Sowden, S. Insights into social perception in autism. J. Neurosci. 2015; 35(23), 8689-8690. 
70. Kaur, M., \& Bhat, A.N. Creative yoga intervention improves motor and imitation skills of children with autism spectrum disorder. Phys. Ther. 99(11), 1520-1534 (2019).

71. Srinivasan, S.M. et al. The effects of rhythm and robotic interventions on the imitation/praxis, interpersonal synchrony, and motor performance of children with autism spectrum disorder (ASD): a pilot randomized controlled trial. Autism Res. Treat. 736516 (2015).

\section{Acknowledgments}

This work was supported by the National Institutes of Health through a shared instrumentation grant awarded to the University of Delaware (Grant \#: 1S10OD021534-01, PI: Bhat), pilot award funding through an Institutional Development Award (IDeA) from the National Institute of General Medical Sciences of the National Institutes of Health (U54-GM104941, PI: BinderMacleod; P20 GM103446, PI: Stanhope) and funding from the Dana Foundation through a Clinical Neuroscience Award (PI: Bhat).

\section{Author Contributions}

W.S was involved in the data collection, data analyses, and manuscript writing. M.C. was involved in the data collection and data analyses. D.T. assisted with the fNIRS spatial registration programs and related graphs. A.B. supervised all aspects of the project including recruitment, screening, student training, data collections, NIRS and IMU data programming, data analysis, and writing and revisions of the manuscript.

\section{Competing Interests}


The funders had no role in study design, data collection and analysis, decision to publish, or preparation of the manuscript. All the authors declare that they have no conflicts of interest. 


\section{Figure Legends}

Figure 1. Movement amplitude (a) and coherence (b) during interpersonal synchrony. * indicates significant differences between the ASD and TD groups

Figure 2. A visual representation of averaged $\mathrm{HbO}_{2}$ concentration during Observation, Solo, Face, and Touch conditions in children with ASD (a) and the TD children (b). $\mathrm{HbO}_{2}$ values on $\mathrm{Y}$-axis range from 0 indicated by blue to 0.18 indicated by red. In Observation and Solo conditions, children with ASD had greater IPL activation than the TD children. During Face condition, children with ASD had lower STS activation than the TD children. Similarly, in Touch condition, children with ASD had lower IFG and STS activation than the TD children.

Figure 3. Group differences in $\mathrm{HbO}_{2}$ concentration during Observation (a), Solo (b), Face (c), and Touch (d) conditions. *indicates significant differences between the ASD and TD groups.

Figure 4. Conditional differences in $\mathrm{HbO} 2$ concentration for the TD children (a) and children with ASD (b). *indicate significant differences between movement conditions

Figure 5. Experimental setup (a) and task sequence (b). Written parental and experimenter permission to use their pictures for this publication has been taken. 


\section{Tables}

Table 1. Correlations between cortical activation and interpersonal synchrony behaviors.

\begin{tabular}{|c|c|c|c|c|}
\hline \multirow[t]{2}{*}{ r-values } & \multicolumn{2}{|c|}{ Sway Amplitude } & \multicolumn{2}{|c|}{ Sway Coherence } \\
\hline & Face & Touch & Face & Touch \\
\hline \multicolumn{5}{|c|}{ TD } \\
\hline \multicolumn{5}{|c|}{ Left hemisphere } \\
\hline MFG & 0.105 & 0.098 & 0.229 & $0.262^{*}$ \\
\hline IFG & 0.213 & 0.141 & $0.263 *$ & 0.179 \\
\hline PCG & 0.210 & 0.211 & $0.287 *$ & -0.019 \\
\hline STS & 0.236 & $0.287 *$ & $0.292 *$ & -0.151 \\
\hline IPL & 0.087 & 0.129 & 0.076 & 0.018 \\
\hline \multicolumn{5}{|c|}{ Right hemisphere } \\
\hline $\mathrm{MFG}$ & 0.212 & 0.117 & 0.249 & $0.267 *$ \\
\hline IFG & $0.326^{*}$ & $0.332 *$ & $0.329 *$ & $0.374 * *$ \\
\hline PCG & $0.463 * *$ & 0.247 & $0.445 * *$ & 0.148 \\
\hline STS & $0.286^{*}$ & 0.087 & $0.354 * *$ & -0.075 \\
\hline IPL & $0.410 * *$ & 0.141 & $0.299 *$ & -0.188 \\
\hline \multicolumn{5}{|l|}{ ASD } \\
\hline \multicolumn{5}{|c|}{ Left hemisphere } \\
\hline MFG & 0.122 & 0.086 & -0.078 & $-0.355^{* *}$ \\
\hline IFG & 0.131 & $0.288 *$ & $-0.266^{*}$ & -0.075 \\
\hline PCG & 0.111 & $0.369 * *$ & -0.231 & $-0.299 *$ \\
\hline STS & $0.346 * *$ & $0.482 * *$ & 0.069 & -0.156 \\
\hline IPL & 0.190 & $0.327 *$ & -0.148 & -0.074 \\
\hline \multicolumn{5}{|c|}{ Right hemisphere } \\
\hline MFG & 0.159 & 0.204 & -0.210 & -0.189 \\
\hline IFG & 0.194 & 0.222 & -0.191 & -0.043 \\
\hline PCG & $0.356 * *$ & $0.423 * *$ & -0.104 & -0.180 \\
\hline STS & $0.258 *$ & 0.233 & $-0.327 *$ & $-0.290 *$ \\
\hline IPL & 0.003 & $0.303^{*}$ & 0.019 & 0.015 \\
\hline
\end{tabular}

$\mathrm{r}$ values are presented in this figure. * indicates $p<0.05$; ** indicates $p<0.01$. Bolded and shaded font indicates $p$ values survived after FDR corrections. 
Table 2. Correlations between cortical activation and social communication performance.

\begin{tabular}{|c|c|c|c|c|c|c|c|c|}
\hline \multirow[t]{2}{*}{ r-values } & \multicolumn{2}{|c|}{$\begin{array}{l}\text { VABS } \\
\text { Communication }\end{array}$} & \multicolumn{2}{|c|}{$\begin{array}{l}\text { VABS } \\
\text { Socialization }\end{array}$} & \multicolumn{2}{|l|}{ SRS } & \multicolumn{2}{|l|}{ ICS } \\
\hline & Face & Touch & Face & Touch & Face & Touch & Face & Touch \\
\hline \multicolumn{9}{|l|}{ TD } \\
\hline \multicolumn{9}{|c|}{ Left hemisphere } \\
\hline MFG & $0.369 * *$ & 0.229 & 0.04 & -0.037 & -0.068 & -0.111 & 0.079 & 0.245 \\
\hline IFG & 0.217 & 0.161 & -0.051 & -0.116 & -0.162 & -0.247 & 0.109 & $0.279 *$ \\
\hline PCG & -0.085 & -0.090 & -0.032 & -0.017 & $0.376 * *$ & 0.128 & $-0.358 * *$ & $-0.264 *$ \\
\hline STS & -0.095 & -0.155 & -0.048 & 0.221 & 0.190 & -0.118 & -0.020 & 0.142 \\
\hline IPL & 0.080 & 0.205 & 0.209 & 0.112 & $0.393 * *$ & 0.143 & -0.115 & -0.084 \\
\hline MFG & $0.286^{*}$ & 0.091 & -0.034 & -0.087 & 0000 & -0.007 & 0.098 & 0154 \\
\hline IFG & 0.215 & 0.115 & -0.201 & $-0.427 *$ & -0.144 & $-0.257 *$ & 0.000 & 0.133 \\
\hline PCG & $0.318 *$ & 0.095 & $-0.332 *$ & -0.168 & $-0.409 * *$ & -0.221 & 0.236 & 0.230 \\
\hline STS & 0.102 & 0.099 & -0.157 & -0.083 & -0.120 & -0.192 & 0.139 & 0.188 \\
\hline IPL & $0.293^{*}$ & 0.082 & $-0.290 *$ & 0.033 & $-0.246^{*}$ & 0.005 & -0.014 & $0.267 *$ \\
\hline \multicolumn{9}{|c|}{ ASD } \\
\hline \multicolumn{9}{|c|}{ Left hemisphere } \\
\hline MFG & 0.011 & $0.284 *$ & 0.113 & 0.205 & -0.069 & -0.162 & 0.219 & 0.186 \\
\hline IFG & -0.152 & 0.163 & -0.094 & 0.098 & 0.158 & 0.008 & 0.101 & $0.436^{* *}$ \\
\hline PCG & -0.108 & $0.275^{*}$ & 0.048 & 0.198 & 0.184 & -0.153 & 0.205 & 0.184 \\
\hline STS & $0.328 * *$ & $0.543 * *$ & 0.136 & $0.345 * *$ & -0.246 & $-0.376 * *$ & 0.178 & -0.058 \\
\hline IPL & 0.172 & $0.479 * *$ & $0.261 *$ & $0.271 *$ & -0.066 & $-0.323^{*}$ & -0.005 & $0.347 * *$ \\
\hline \multicolumn{9}{|c|}{ Right hemisphere } \\
\hline MFG & 0.032 & 0.193 & 0.083 & 0.129 & -0.137 & -0.096 & 0.156 & 0.037 \\
\hline IFG & 0.093 & $0.331 * *$ & 0.208 & $0.287 *$ & -0.026 & -0.153 & -0.038 & 0.017 \\
\hline PCG & $0.410 * *$ & $0.555 * *$ & $0.284 *$ & $0.454 * *$ & $-0.285^{*}$ & $-0.354 * *$ & 0.171 & 0.184 \\
\hline STS & $0.303^{*}$ & 0.204 & 0.192 & 0.073 & -0.164 & 0.028 & -0.003 & -0.002 \\
\hline IPL & -0.029 & 0.224 & 0.140 & 0.068 & -0.078 & -0.020 & 0.064 & 0.127 \\
\hline
\end{tabular}

$\mathrm{r}$ values are presented in this table. *indicates $p<0.05 ; * *$ indicates $p<0.01$. Bolded and shaded font indicates $p$ values survived for FDR corrections. 
Table 3. Demographic information and the results of social communication questionnaires in ASD and TD groups. Means and standard errors (SE) provided.

\begin{tabular}{|l|l|l|}
\hline Characteristics & $\begin{array}{l}\text { ASD group }(\mathrm{n}=15) \\
\text { Mean } \pm \mathrm{SE}\end{array}$ & $\begin{array}{l}\text { TD group }(\mathrm{n}=15) \\
\text { Mean } \pm \mathrm{SE}\end{array}$ \\
\hline Age & $12.7 \pm 0.8$ & $12.5 \pm 0.8$ \\
\hline Sex & $12 \mathrm{M}, 3 \mathrm{~F}$ & $10 \mathrm{M}, 5 \mathrm{~F}$ \\
\hline Ethnicity & $1 \mathrm{~A}, 1 \mathrm{AC}, 13 \mathrm{C}$ & $2 \mathrm{~A}, 13 \mathrm{C}$ \\
\hline VABS-II $(\%)$ & $8.5 \pm 3.6^{*}$ & $58.4 \pm 7.3$ \\
Communication $(\%)$ & $12.8 \pm 4.6^{*}$ & $59.9 \pm 6.8$ \\
Daily living (\%) & $18.5 \pm 7.0^{*}$ & $58.4 \pm 8.2$ \\
Socialization (\%) & $5.3 \pm 2.1^{*}$ & $60.0 \pm 7.9$ \\
\hline SRS (T scores) & $77.7 \pm 2.7^{*}$ & $44.87 \pm 1.62$ \\
\hline ICS & $4.0 \pm 0.3^{*}$ & $5.7 \pm 0.2$ \\
\hline Handedness & $13 \mathrm{R}, 2 \mathrm{~L}$ & $14 \mathrm{R}, 1 \mathrm{~L}$ \\
Coren's score & $31.2 \pm 1.2$ & $34.0 \pm 1.6$ \\
\hline
\end{tabular}

VABS-II $=$ Vineland Adaptive Behavior Scale $-2^{\text {nd }}$ Edition; SRS $=$ Social Responsiveness Scale ICS $=$ Interpersonal Communication Scale; $\mathrm{M}=$ Male, $\mathrm{F}=$ Female; $\mathrm{C}=$ Caucasian, $\mathrm{A}=$ Asian, $\mathrm{AC}=$ AsianCaucasian. $\mathrm{R}=$ right-handed, $\mathrm{L}=$ left-handed. $*$ indicates significant differences between children with and without ASD $(p<0.05)$. 
Figures

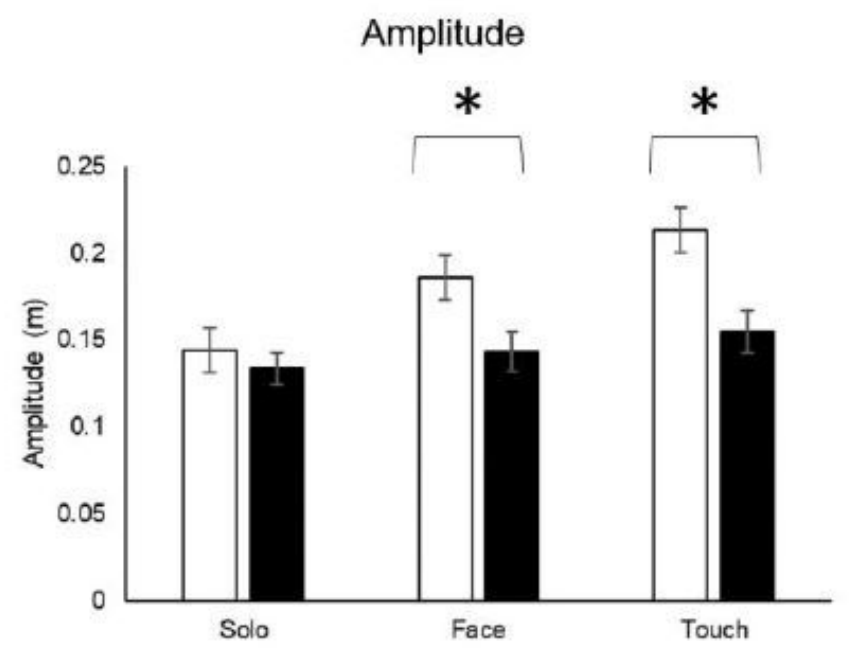

(a)

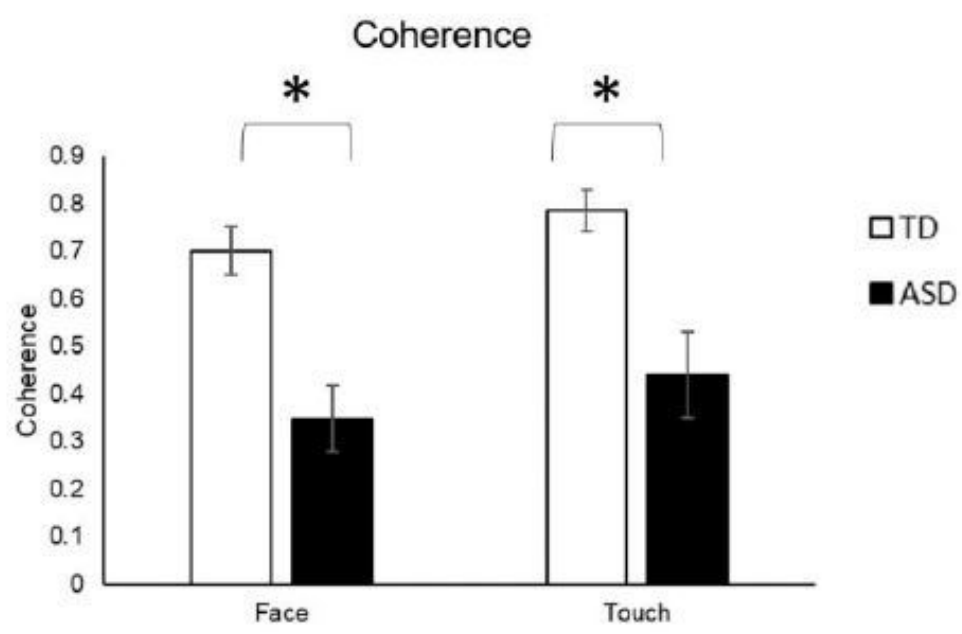

(b)

\section{Figure 1}

Movement amplitude (a) and coherence (b) during interpersonal synchrony. * indicates significant differences between the ASD and TD groups

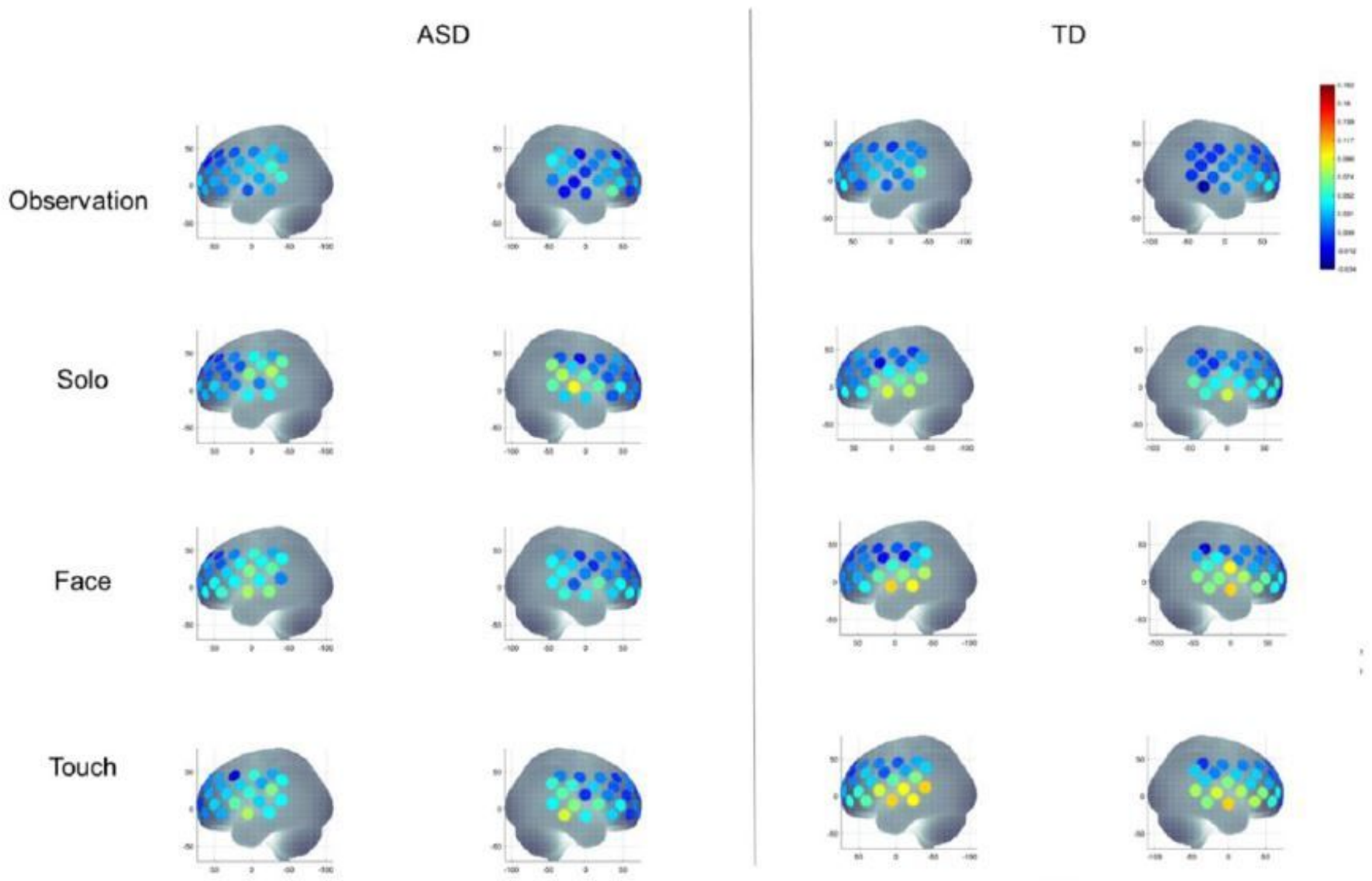

(a)

(b) 
Figure 2

A visual representation of averaged $\mathrm{HbO} 2$ concentration during Observation, Solo, Face, and Touch conditions in children with ASD (a) and the TD children (b). HbO2 values on Y-axis range from 0 indicated by blue to 0.18 indicated by red. In Observation and Solo conditions, children with ASD had greater IPL activation than the TD children. During Face condition, children with ASD had lower STS activation than the TD children. Similarly, in Touch condition, children with ASD had lower IFG and STS activation than the TD children.

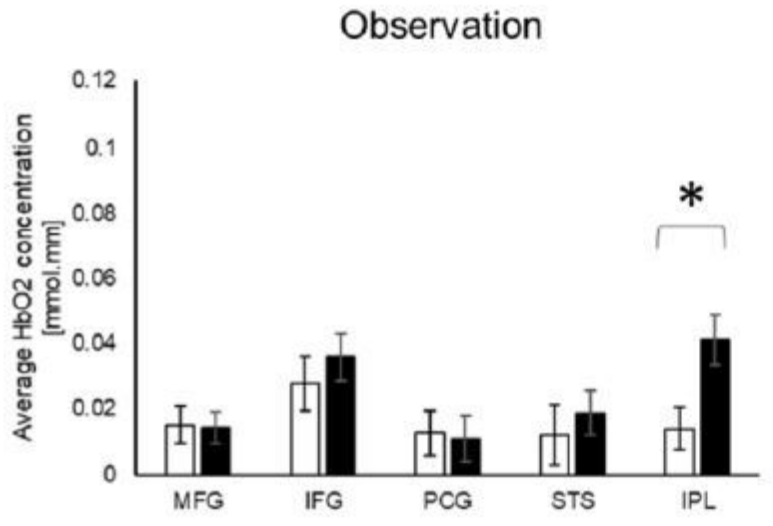

(a)

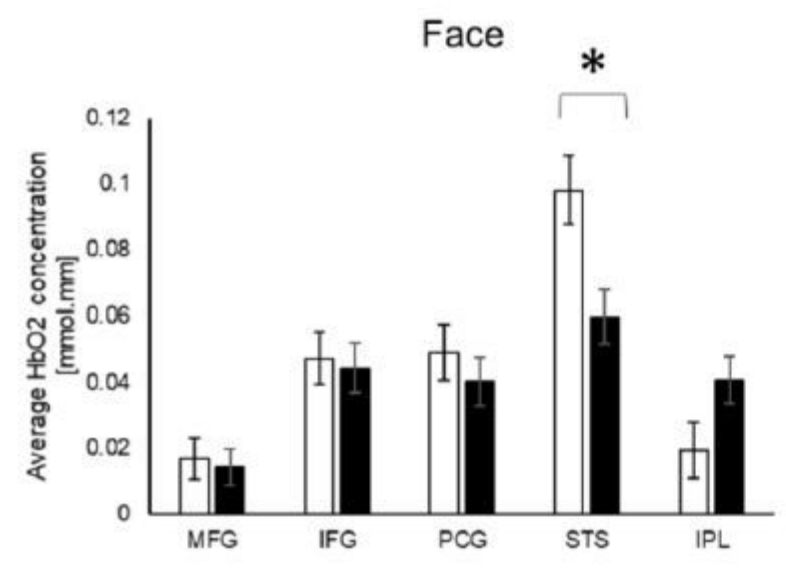

(c)

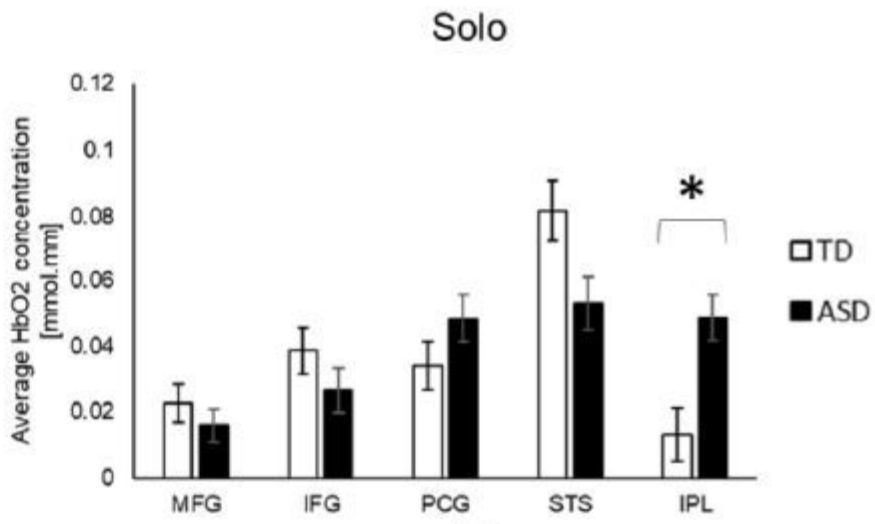

(b)

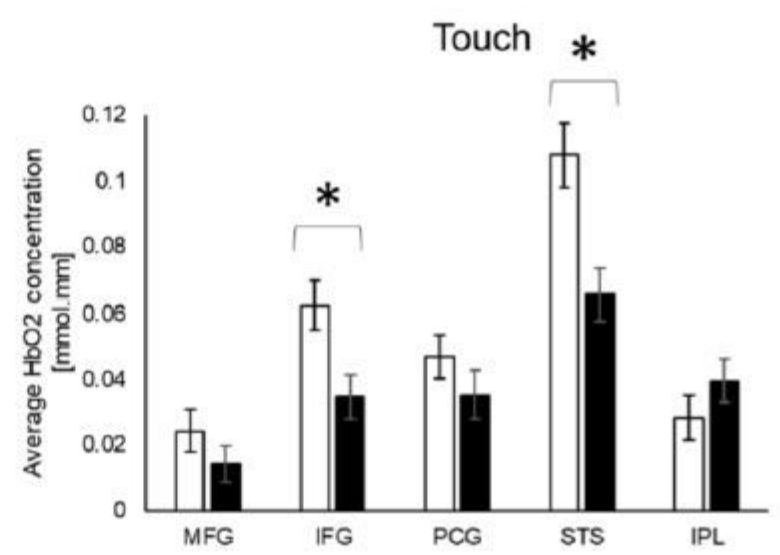

(d)

Figure 3

Group differences in HbO2 concentration during Observation (a), Solo (b), Face (c), and Touch (d) conditions. *indicates significant differences between the ASD and TD groups. 


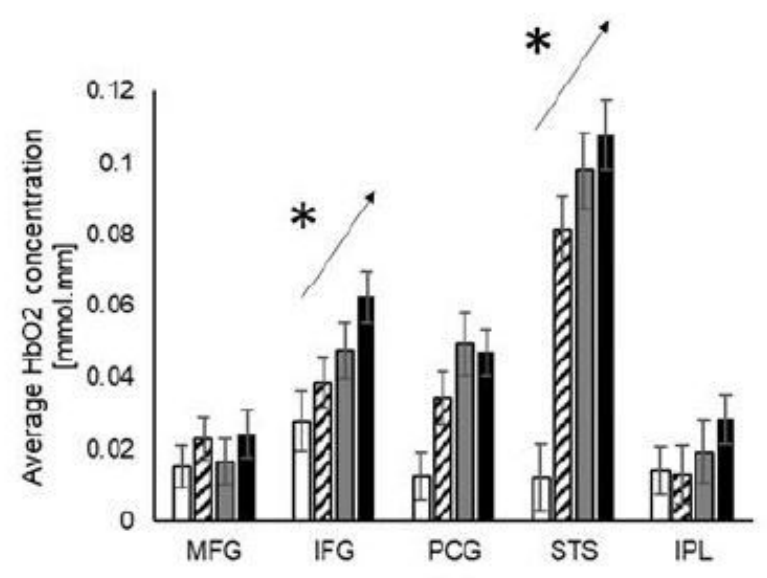

(a)

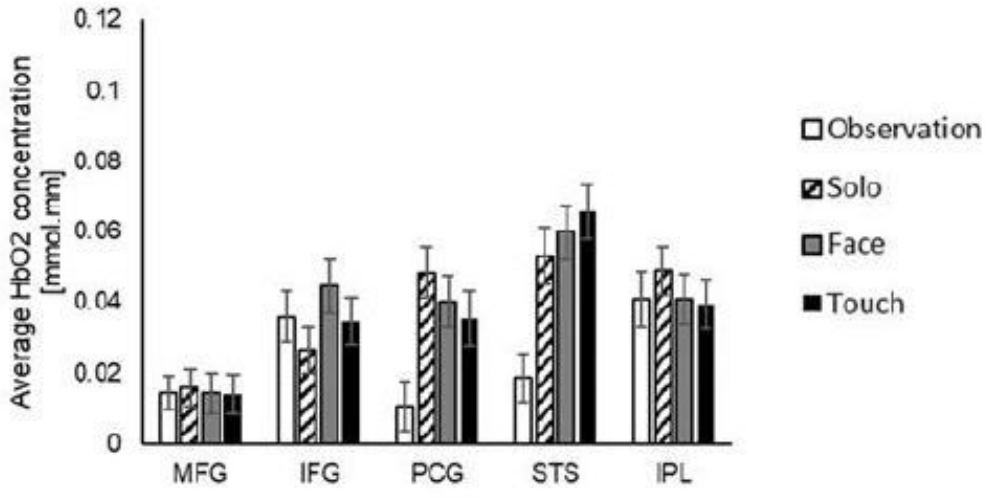

(b)

\section{Figure 4}

Conditional differences in $\mathrm{HbO} 2$ concentration for the TD children (a) and children with ASD (b). *indicate significant differences between movement conditions

\section{Experimental Procedures}
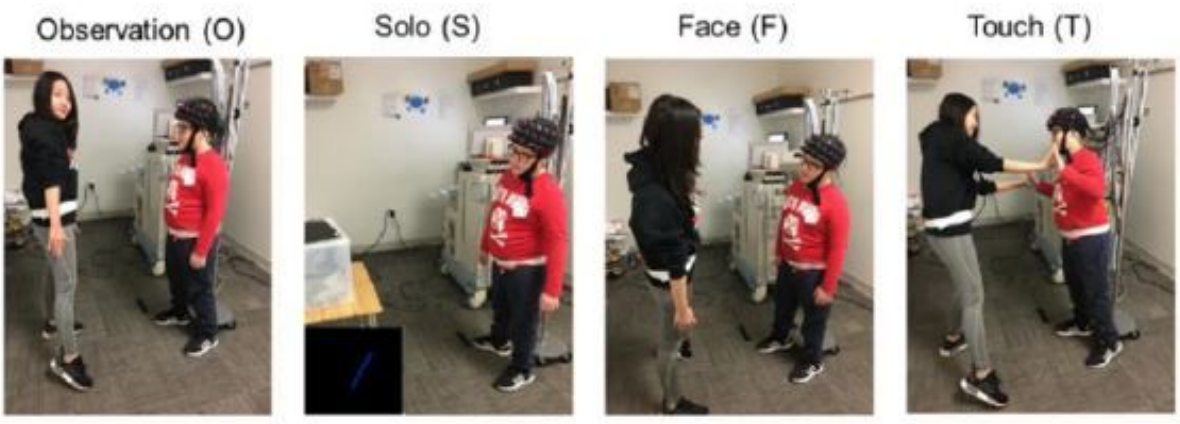

(a)

Trial Sequence in E-Prime

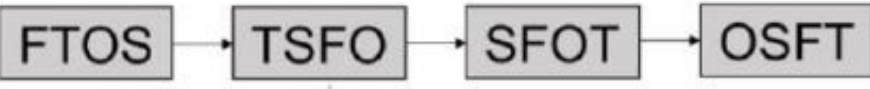

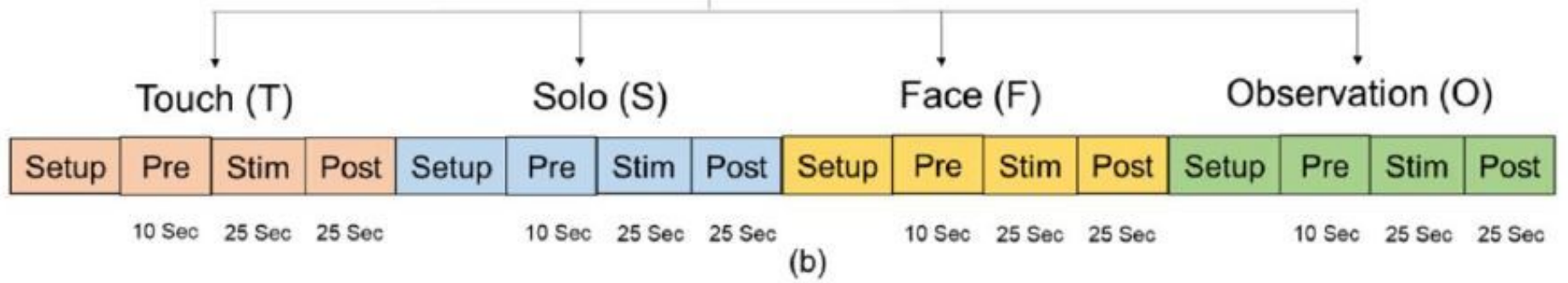


Figure 5

Experimental setup (a) and task sequence (b). Written parental and experimenter permission to use their pictures for this publication has been taken.

\section{Supplementary Files}

This is a list of supplementary files associated with this preprint. Click to download.

- POSSuppMaterialScientificReport.pdf 